\title{
Meningioma: A Clinicopathological Correlation
}

\author{
Asha Shenoy ${ }^{1}$, Shalaka Khade ${ }^{2 *}$ and Ramesh Waghmare ${ }^{2}$ \\ ${ }^{1}$ Department of Pathology, Seth G. S. Medical College \& K.E.M. Hospital, Mumbai, India \\ ${ }^{2}$ Department of Pathology, Topiwala National Medical College \& B.Y.L. Nair Hospital, Mumbai, India
}

\section{ABSTRACT}

Background: This study aimed at classifying meningioma and its variants based on histology, and study its clinicopathological correlation This analytical study conducted in a tertiary referral centre included clinical presentation, radiological features and histopathological correlation of meningioma. The grading system of meningioma is based on histological features. Difference in subjective assessment can hamper this grading system. A continuous revision of the histopathology of meningiomas and taking into account precise definitions of features such as small cell changes, hypercellularity, sheeting, necrosis, and mitotic count is necessary to improve the accuracy for diagnosis grading of these tumours.

Methods: A retrospective and prospective study of meningiomas was carried out in the department of Pathology at a tertiary care hospital over a period of 5 years (July 2011 to June 2016). Clinical presentation, radiological features and histological findings were taken into consideration.

Result: Of the 277 cases of lesions of central nervous system, 63 cases were diagnosed as meningioma, the incidence being $22.74 \%$. Most these cases were in the 4 th to 6 th decade of life $(71.42 \%)$. The overall male to female ratio was $1: 2.3$. The commonest clinical feature was headache $(58.73 \%)$ followed by limb weakness (33.33\%). The most common location was cerebral hemisphere (49.20\%). There were 7 cases of spinal meningioma. Most of the meningiomas were hyperdense (60.8\%) on CT and iso to hypointense (90\%) on MRI.Most of the cases were Grade I meningiomas $(88.89 \%)$, meningothelial and transitional being the most common histological subtypes. Two of the grade II meningioma cases showed presence of brain invasion.

Conclusion: Histopathological examination is an imperative tool for confirmatory diagnosis due to the diverse histological variants. Also, the prognosis of the disease depends on histopathological grading of the lesion.

\section{Keywords: Meningioma, Psammomatous Meningioma}

\section{Introduction}

Meningioma is a neoplasm arising from the arachnoidal cap cells in the meningeal coverings of the spinal cord and brain. These are the most common benign intracranial tumours and account for about $13-26 \%$ of all primary brain neoplasms ${ }^{[1]}$. These are located in both intracranial and intraspinal region. The peak incidence is in middle aged patients and there is a marked female predominance with the male: female ratio being approximately $1: 2^{[2]}$. Meningiomas are generally benign, slow growing tumours; but atypical forms can also occur.

Various classifications were put forth to classify meningiomas which have undergone many changes. Currently WHO 2016 classification of Meningiomas is being followed. Histological grading of meningioma has prognostic and therapeutic implications. Features such as small cell changes, hypercellularity, sheeting, necrosis, mitotic count and brain invasion are the histological parameters that have a role in grading of meningiomas. According to this grading system, meningioma is divided into three grades- benign (Grade I), atypical (Grade II) and anaplastic (Grade III).

This is an analytical study (retrospective and prospective) conducted in a tertiary referral centre. The aim of this study is to classify meningioma and its variants based on histology, and study its clinicopathological correlation with respect to clinical presentation, radiological features and histopathological appearance.

\section{Material and Methods}

A retrospective and prospective study of meningiomas was carried out in the department of Pathology at a tertiary care hospital over a period of 5 years (July 2011 to June 2016). Ethical committee clearance was obtained before the commencement of the study. Clinical data and imaging findings of the patient were obtained from patients' records available in the Medical Records Department for the retrospective study. The Haemotoxylin and Eosin $(\mathrm{H} \&$ E) stained slides of the cases were reviewed keeping in view the histological features. Sections were recut from the paraffin blocks available in the Pathology department 
wherever needed. For the prospective study, biopsy tissue was fixed overnight in $10 \%$ buffered formalin and submitted for processing. Paraffin sections were cut 4 to 6 microns in thickness and routine H\&E staining was performed. Special stains were performed whenever necessary. Radiological imaging and operative findings were scrutinized to know the anatomical location of the tumour. Histopathology findings were taken into consideration for classification as per WHO 2016 classification of Meningiomas. As immunohistochemistry was not available at our institution, patients were referred for IHC to oncology institution.

\section{Results}

Over a period of 5 years, 277 cases of lesions of central nervous system were found out of which meningioma cases were 63 (22.74\%). Maximum number of cases was found in the $4^{\text {th }}$ to 6 th decade of life $(71.42 \%)$. The youngest patient in our study was 6 years old male child, while the oldest was 65 years old. All the grade II meningioma cases noted were in the $5^{\text {th }}$ to $7^{\text {th }}$ decade of life. We found female predominance in our study, 44 out of 63 cases being female and 19 being male. Hence, the Male: Female ratio is 1:2.3 (Table 1)

Most common symptom was headache, followed by limb weakness in both grade I and grade II cases. Headache was present in 37 out of 63 cases and limb weakness in 21 cases. Other symptoms included giddiness, convulsion, vomiting and diminution of vision. The duration of symptoms ranged from two days to 5 years overall.
About $69.8 \%$ of meningioma cases were supratentorial and rest $30.15 \%$ were infratentorial. The most common location of meningioma was found to be the cerebral convexity, and in this frontal was the most common. There were 7 cases of spinal meningioma. (Table 2).

Meningiomas demonstrated iso to hypointensity in $90 \%$ (36) cases on T1 weighted MRI, and were hyperdense in $60.8 \%$ (14) cases on CT scan. Most of them (92\%) showed homogenous enhancement. Perilesional edema was present in $74.6 \%$ (47) cases. All 7 cases of grade II meningioma demonstrated perilesional edema. 6 cases showed areas of calcification within $(9.5 \%)$ and hyperostosis was seen in one case. $88.89 \%$ cases belonged to WHO grade I, and $11.11 \%$ cases belonged to WHO grade II. There were no cases of WHO grade III. Females out numbered males in both grade I and grade II meningiomas. Most of the cases were WHO grade I, out of which the most common subtypes were meningothelial and transitional comprising of $28.57 \%$ (18) each. This was followed by psammomatous type comprising 15.875 (10). Maximum numbers of grade II meningioma ( 6 out 7 ) were seen in female. Among the intracranial cases, meningothelial and transitional variants were most common (Figure 1). In intraspinal cases, psammomatous meningioma was predominant. Other grade I subtypes included angiomatous, fibrous, microcystic and secretory meningioma (Figure 2 and 3). All 7 cases of atypical meningioma occurred in 5th, 6th and 7 th decade (Table 3). Brain invasion was observed in 2 cases. (Table 4, Figure 3). MIB labeling index was advised in all cases.

Table 1: Age and Gender Distribution.

\begin{tabular}{|c|c|c|c|c|}
\hline \multirow{2}{*}{ AGE } & \multicolumn{2}{|c|}{ GENDER } & \multirow{2}{*}{ TOTAL } & \multirow{2}{*}{ PERCENTAGE } \\
\cline { 2 - 5 } & MALE & FEMALE & 1 & $0.01 \%$ \\
\hline $0-10$ & 1 & 0 & 3 & $0.04 \%$ \\
\hline $11-20$ & 1 & 2 & 6 & $0.09 \%$ \\
\hline $21-30$ & 3 & 3 & 17 & $26.98 \%$ \\
\hline $31-40$ & 5 & 12 & 11 & $17.46 \%$ \\
\hline $41-50$ & 3 & 12 & 8 & $26.98 \%$ \\
\hline $51-60$ & 5 & 7 & $\mathbf{6 3}$ & $12.69 \%$ \\
\hline TOTAL & 1 & 44 & $100 \%$ \\
\hline
\end{tabular}

Table 2: Location Wise Distribution of Meningioma .

\begin{tabular}{|c|c|c|c|}
\hline \multicolumn{2}{|c|}{ LOCATION } & CASES & PERCENTAGE \\
\hline \multirow{4}{*}{ Cerebral convexity } & Frontal & 11 & $17.46 \%$ \\
\cline { 2 - 4 } & Fronto-parietal & 3 & $4.76 \%$ \\
\cline { 2 - 4 } & Frontotemporoparietal & 2 & $3.17 \%$ \\
\cline { 2 - 4 } & Parietal & 7 & $11.11 \%$ \\
\cline { 2 - 4 } & Parieto-temporal & 3 & $4.76 \%$ \\
\cline { 2 - 4 } & Parieto-occipital & 3 & $4.76 \%$ \\
\hline
\end{tabular}




\begin{tabular}{|c|c|c|}
\hline \multicolumn{1}{|c|}{ LOCATION CASES } & PERCENTAGE \\
\hline \multirow{2}{*}{$\begin{array}{c}\text { Cemporal } \\
\text { Occipital }\end{array}$} & 1 & $1.58 \%$ \\
\hline Cerebellopontine angle & 1 & $1.58 \%$ \\
\hline Sphenoid wing & 7 & $11.11 \%$ \\
\hline Posterior fossa & 6 & $9.52 \%$ \\
\hline Intraventricular & 3 & $4.76 \%$ \\
\hline Parafalcine/Parasagittal & 2 & $3.17 \%$ \\
\hline Suprasellar & 2 & $3.17 \%$ \\
\hline Cerebellar & 2 & $3.17 \%$ \\
\hline Retrobulbar & 1 & $1.58 \%$ \\
\hline Craniovertebral & 1 & $1.58 \%$ \\
\hline Spinal & 1 & $1.58 \%$ \\
\hline Total & 7 & $11.11 \%$ \\
\hline
\end{tabular}

Table 3: Histological Distribution of Meningioma Subtypes.

\begin{tabular}{|c|c|c|c|}
\hline \multicolumn{2}{|c|}{ HISTOLOGICAL SUBTYPES } & \multirow{2}{*}{$\begin{array}{c}\text { NUMBER OF CASES } \\
18\end{array}$} & \multirow{2}{*}{$\begin{array}{c}\text { PERCENTAGE (\%) } \\
28.57 \%\end{array}$} \\
\hline \multirow{9}{*}{ Grade I } & Meningothelial & & \\
\hline & Transitional & 18 & $28.57 \%$ \\
\hline & Psammomatous & 10 & $15.87 \%$ \\
\hline & Angiomatous & 6 & $9.52 \%$ \\
\hline & Fibrous & 2 & $3.17 \%$ \\
\hline & Microcystic & 1 & $1.58 \%$ \\
\hline & Secretory & 1 & $1.58 \%$ \\
\hline & Lymphoplasmacyte-rich & 0 & $0 \%$ \\
\hline & Metaplastic & 0 & $0 \%$ \\
\hline \multirow{3}{*}{ Grade II } & Atypical & 5 & $7.93 \%$ \\
\hline & Chordoid & 2 & $3.17 \%$ \\
\hline & Clear cell & 0 & $0 \%$ \\
\hline \multirow{4}{*}{ Grade III } & Anaplastic & 0 & $0 \%$ \\
\hline & Rhabdoid & 0 & $0 \%$ \\
\hline & Papillary & 0 & $0 \%$ \\
\hline & Total & 63 & $100 \%$ \\
\hline
\end{tabular}

Table 4: Histological Features.

\begin{tabular}{|c|c|c|}
\hline HISTOLOGICAL FEATURES & NUMBER OF CASES & PERCENTAGE (\%) \\
\hline Mitosis & 18 & $28.57 \%$ \\
\hline Necrosis & 8 & $12.69 \%$ \\
\hline Psammoma bodies & 28 & $44.44 \%$ \\
\hline Brain invasion & 2 & $3.17 \%$ \\
\hline Hypercellularity & 4 & $6.34 \%$ \\
\hline Patternless growth & 0 & $0 \%$ \\
\hline High nucleus:cytoplasmic ratio & 3 & $4.76 \%$ \\
\hline Macronucleoli & 7 & $11.11 \%$ \\
\hline Nuclear pleomorphism & 11 & $17.46 \%$ \\
\hline
\end{tabular}




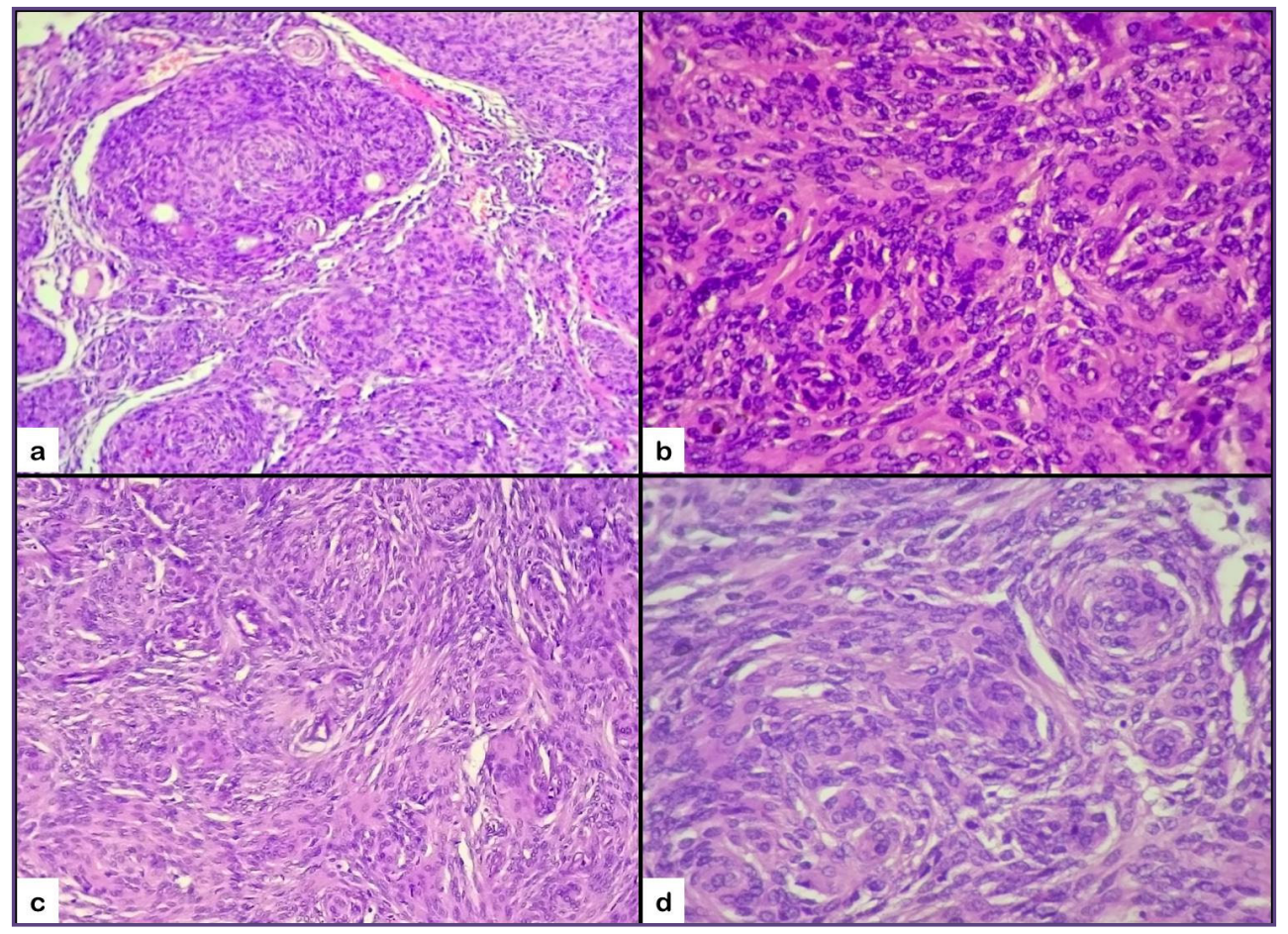

Fig. 1: (a) Meningothelial cells arranged in lobules in meningothelial meningioma (H\&E, 100X) (b) Meningothelial meningioma showing cells arranged in syncytium (H\&E, 400X) (c) Transitional meningioma showing vaguely lobular and fascicular arrangement of cells (H\&E,100X) (d) Transitional meningioma highlighting cells arranged in whorls (H\&E,400X).

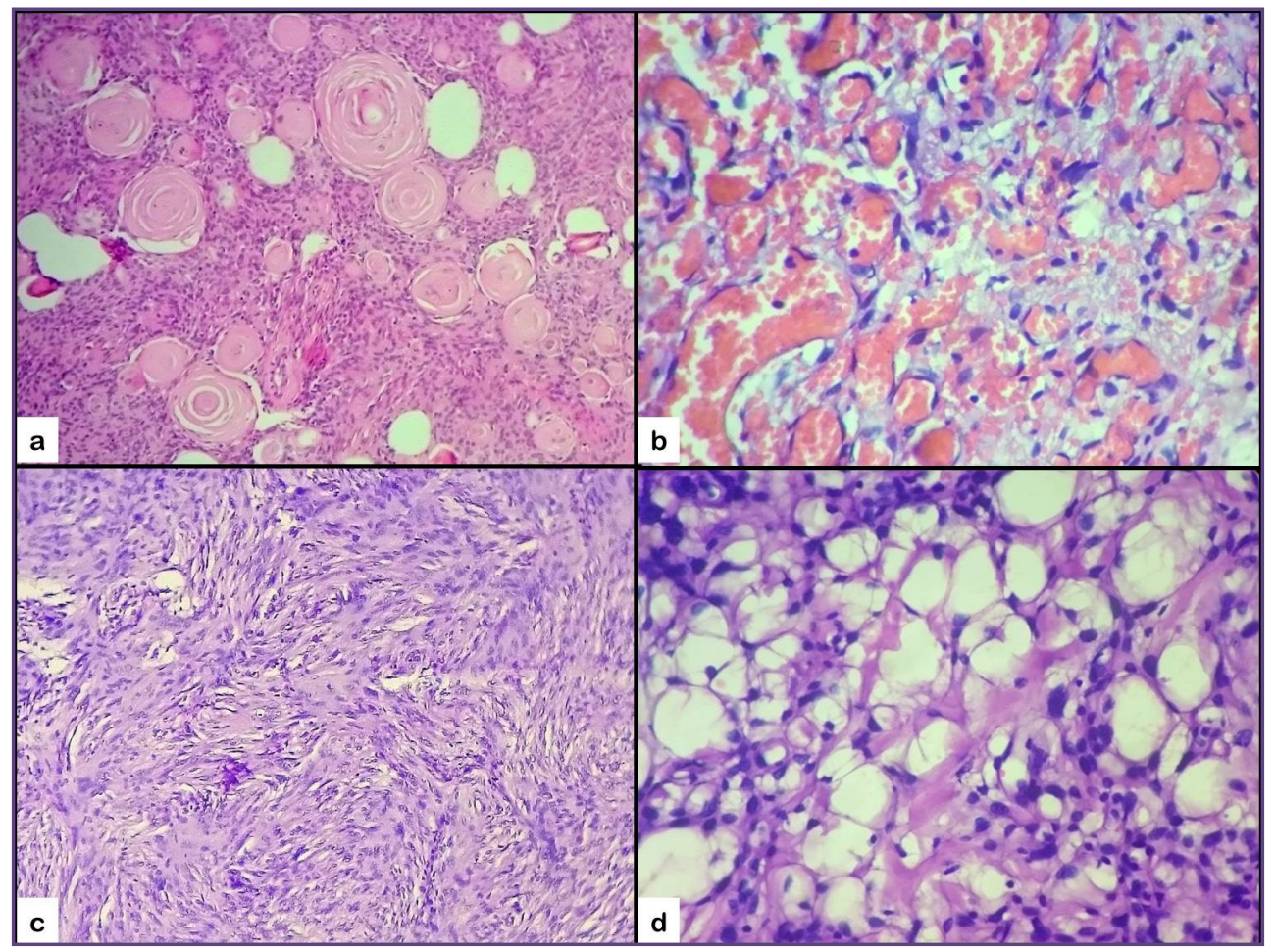

Fig. 2: (a) Psammomatous meningioma showing predominant psammoma bodies (H\&E,100X) (b) Angiomatous meningioma showing numerous vascular channels (H\&E,400X) (c) Fibrous meningioma showing cells in fascicles and bundles (H\&E,100X) (d) Microcystic meningioma showing numerous cystic spaces lined by stellate-shaped meningothelial cells (H\&E,400X). 


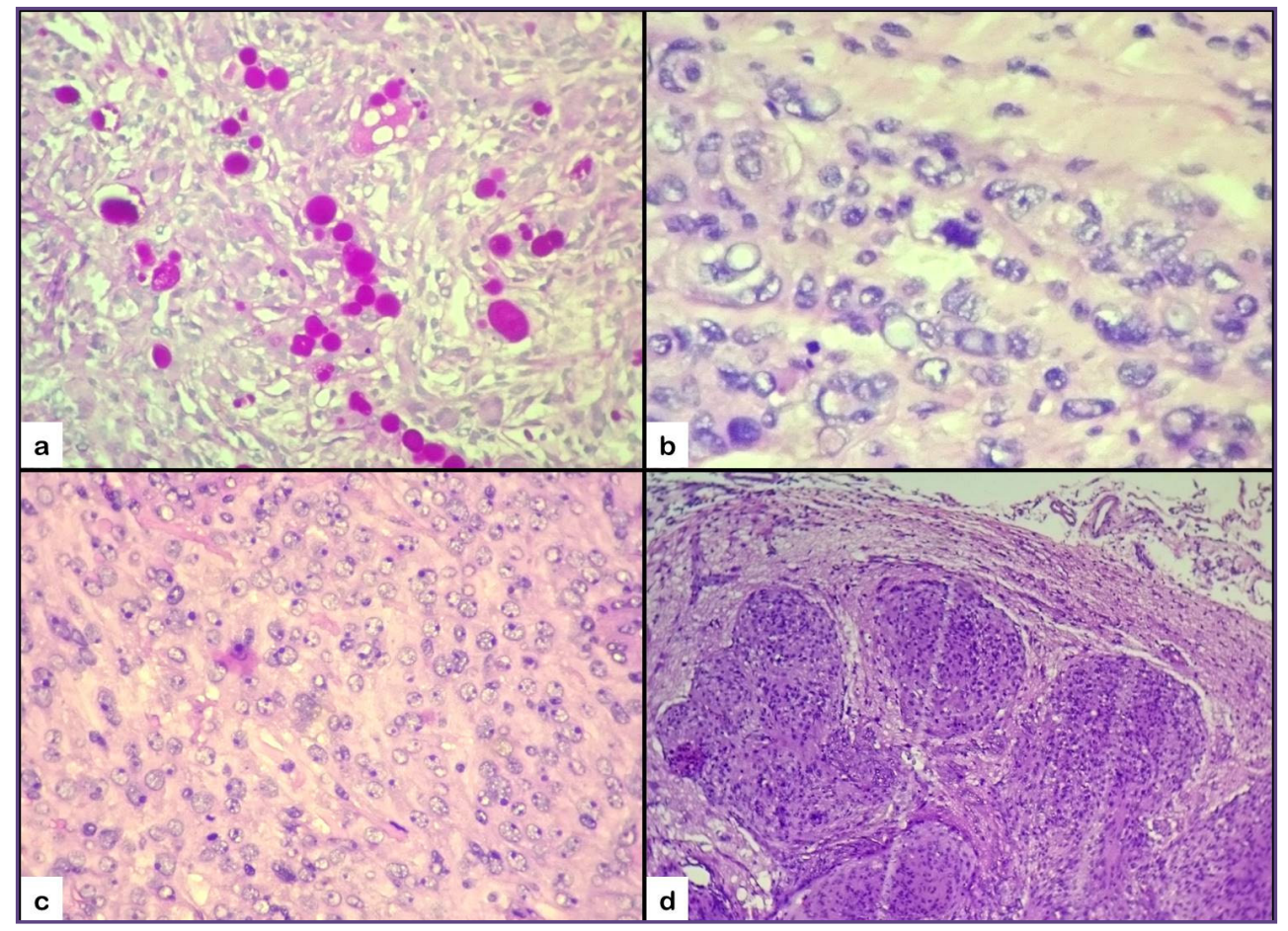

Fig. 3: (a) Eosinophilic material in secretory meningioma showing PAS positivity (PAS,400X) (b) Photomicrograph showing mitotic figures (H\&E,400X) (c) Photomicrograph showing tumour cells with macronucleoli (H\&E,400X) (d) Brain invasionTongue like protrusion of tumour cells infiltrating brain parenchyma without intervening layer of leptomeninges (H\&E, 100X).

\section{Discussion}

Meningiomas are the primary non-glial tumours of the central nervous system accounting for about $13-26 \%$ of all the intracranial tumours. These are the most common extra-axial neoplasms. WHO classification of tumours of the Central Nervous System has defined meningiomas as "meningothelial (arachnoid) cell neoplasms, typically attached to the inner surface of the dura mater"[3].Clinically, patients with meningioma have varied symptoms and mostly present with neurological signs and symptoms caused by pressure effect of tumour on adjacent neural tissue. Few percentages of meningiomas are asymptomatic and are incidental findings at autopsy. Meningiomas have heterogenous histological picture and are divided into three grades according to WHO classification. Most of the meningiomas are benign, but few have atypical and malignant features. Higher grade of meningioma is associated with increased chances of recurrence and biologically aggressive behaviour.

In the present study, 45 cases $(71.42 \%)$ of meningiomas were encountered in fourth to sixth decade. The youngest patient in our study was a 6 year old male child, while the oldest patient was 65 years old. The mean age in our study was 44.74 years. These results were in concordance with the study conducted by Desai et al ${ }^{[4]}$ which showed maximum incidence in the fourth and fifth decade. Meningiomas are rare in children ${ }^{[5]}$. Our study also found similar results. In our study, we found only one pediatric case who was a 6 year old male child.

Out of the 63 cases in our study, 19 were male and 44 were female. The male: female ratio was found to be $1: 2.3$ with a clear female predominance. Most of the studies in literature mention a female predominance in meningioma. Our results were comparable to those found in a study conducted by Backer et al ${ }^{[6]}$ showing the male: female ratio to be 1:3. Female preponderance may be explained by the progesterone dependent tumour growth as documented in various studies ${ }^{[7]}$. There were 7 cases of intraspinal meningioma, all of which were female patients. Many studies have suggested that spinal meningiomas are 2-3 times more common in female than in male ${ }^{[8]}$.

The clinical signs and symptoms of the patient depend on location of tumour, its size and effect on surrounding. The most common presenting symptom in our study was headache, which was seen in 37 out of 63 cases $(58.73 \%)$. Headache was seen in 33 cases in grade I meningioma and 4 cases of grade II meningioma. This was followed by limb weakness $(33.33 \%)$, convulsion $(23.80 \%)$, giddiness $(19.04 \%)$, vomiting $(17.46 \%)$, and diminution of vision $(11.11 \%)$. Moradi et al ${ }^{[9]}$ in his study on meningioma found 
headache/vertigo as the most common symptom seen in $66.7 \%$ cases. These results corroborated with those found in our study. No significant difference was noted in the symptoms of grade I and grade II meningiomas.

44 out of $63(69.85 \%)$ meningioma cases in our study were supratentorial in location, while the rest 19 cases (30.15\%) were infratentorial. Many studies predominantly found meningioma in supratentorial location ${ }^{[10-12]}$. In our study, 56 cases $(88.89 \%)$ were intracranial whereas 7 cases $(11.11 \%)$ were of spinal meningioma. These results were similar to those found by Patil et al ${ }^{[13]}$ who noted intracranial and spinal meningioma to be $90.81 \%$ and $9.19 \%$ respectively. Cerebral convexity was involved in 31 out of 63 cases $(49.20 \%)$ in this study. These results were in concordance with the studies done by Backer et al ${ }^{[6]}$ and Shri Lakshmi et al ${ }^{[12]}$ in which cerebral convexities was involved in $39.3 \%$ and $37.27 \%$ cases respectively. In the cerebral convexities, 11 cases were seen to involve frontal lobe, followed by 7 cases in parietal lobe. Following cerebral convexities, other common locations included cerebellopontine angle and sphenoid wing which constituted $7(11.11 \%)$ and 6 $(9.52 \%)$ cases respectively. A study conducted by Moradi et al ${ }^{[9]}$ demonstrated cerebral convexities as the most common site $(66.2 \%)$, followed by sphenoid ridge $(17.2 \%)$ and cerebellopontine angle $(7.7 \%)$ of all the meningioma cases. These results are in accordance with our study. Intraventricular region is a rare site accounting for about only $0.7-2 \%$ of intracranial meningiomas ${ }^{[14]}$. We also found one case each at cerebellar, retrobulbar and craniovertebral region (foramen magnum) (1.58\%). This is comparable to the study done by Shrilakshmi et al ${ }^{[12]}$ where $1.82 \%$ of meningioma cases were located at craniovertebral region. All the intraspinal meningiomas in our study were located in the thoracic segment. Similar observations noted by Galgano et al ${ }^{[8]}$, indicated thoracic segment as the most common spinal site for intraspinal meningiomas.

In our study, MRI findings were available in 40 cases. Out of these, 36 cases $(90 \%)$ showed iso to hypointensity on T1 weighted imaging out of which 4 cases were of grade II. Similar findings were demonstrated by Gangadhar et al ${ }^{[11]}$ who found isointensity in majority of cases. CT scan findings were available in 23 cases, out of which 14 cases (60.8\%) showed hyperdensity, out of which 3 cases were of grade II meningioma. Similar findings were noticed by Mary et al ${ }^{[15]}$ showing hyperdensity on $\mathrm{CT}$ in $87 \%$ cases. In our study, 58 out of 63 cases of meningioma (92.06\%) showed homogenous enhancement on radioimaging. This was consistent with the study done by Gangadhar et al ${ }^{[11]}$ who demonstrated homogenous enhancement of the mass in $82.61 \%$ cases. Presence of perilesional edema is frequently found in meningiomas. Our study showed 37 cases out of 63 (58.73\%) having perilesional edema, which was comparable to results of Kim et al ${ }^{[16]}$ who demonstrated perilesional edema in $58.1 \%$ cases. All 7 cases of grade II meningioma demonstrated perilesional edema. Peritumoral edema may cause elevated intracranial pressure and may be associated with increased likelihood of developing symptoms. There are many theories stating the development of peritumoral edema related to meningioma. One theory postulates compression of brain with development of ischaemia and edema; whereas another states occlusion of major cerebral veins and dural sinuses as the cause. However, exact cause of peritumoral brain edema is not known. Hyperostosis and calcification are best appreciated on CT. Hyperostosis was present in only one case (1.58\%) which was meningothelial subtype, whereas calcification was noted in 6 cases $(9.5 \%)$ comprising of 5 grade I and one grade II cases, which is comparable to study conducted by Mary et al ${ }^{[15]}$ in which calcification was seen in $11 \%$ cases and hyperostosis in 13\%. Dural tail sign, which is distinctive but not specific for meningioma, was present in 19 cases $(30.15 \%)$ and CSF cleft sign was present in 9 cases $(14.28 \%)$ in our study. Only one case each of grade II meningioma demonstrated dural tail sign and CSF cleft sign. Gangadhar et al ${ }^{[1]}$ in his study on meningioma found dural tail sign in $23.9 \%$ cases and CSF cleft sign in $52.17 \%$ cases.

Histopathology is necessary not only for the diagnosis of meningioma, but also has a prognostic implication. Also, the current grading system of meningioma is based on the histological features. Meningiomas are known to have a considerable heterogeneity, due to which the classification scheme has undergone repeated revisions. Although most of the meningiomas are benign, histologically distinct subtypes are associated with aggressiveness. These are generally slow growing tumours, but some may recur even after resection. Recurrence is associated with both incomplete resection and histological aggressiveness. The association between histological grading and prognosis is formalized by the WHO grading system. For histological classification and grading; tissue pattern, cell morphology, mitosis, psammoma bodies, high nuclear: cytoplasm ratio, necrosis, macronucleoli and brain invasion are taken into consideration. Presence of high mitotic index and brain invasion are sufficient to assign higher grade for meningioma and such meningiomas show aggressive behaviour.

Out of the total 63 meningioma cases, 56 belonged to grade I $(88.89 \%)$ and 7 cases $(11.11 \%)$ belonged to grade II. We did not find any Grade III cases during our study period. Gadgil et al ${ }^{[17]}$, in his study on meningioma noted $85.6 \%$ grade I, $11.5 \%$ grade II and $2.9 \%$ grade III. A study by 
Desai et al ${ }^{[4]}$ found the percentage of grade I, grade II and grade III meningiomas to be $90 \%, 8 \%$ and $2 \%$ respectively. Our results were consistent with the above studies and many other studies in literature. In all these studies, most common type was benign WHO grade I meningioma.

In our study, there were total 56 cases of grade I meningioma out of the total 63 meningioma cases. Out of these, 18 were male $(32.14 \%)$ and 38 were female $(67.85 \%)$. There was a female preponderance in Grade I meningioma and the male: female ratio was $1: 2.1$. In the study conducted by Backer et al ${ }^{[6]}$, there were 135 cases of grade I meningioma, out of which 107 cases were female $(79.3 \%)$ and 28 were male $(20.7 \%)$ with a male: female ratio of $1: 3.8$.

Meningothelial and transitional meningioma were the most common subtypes in grade I, each composing of 18 cases out of the total 63 cases $(28.57 \%)$. This was followed by psammomatous meningioma which constituted 10 cases (15.87\%). Meningothelial variant was the most common variant in Moradi et al ${ }^{[9]}$, Jindal et al ${ }^{[18]}$ and Patil et al ${ }^{[13]}$ accounting for $33.7 \%, 50 \%$ and $43.67 \%$ respectively while transitional variant was commonest in the studies done by Backer et al ${ }^{[6]}$ constituting $39.8 \%$.One case of transitional meningioma was found in the intraspinal location while the other 17 cases had intracranial location.

Psammomatous variant is the more common histological type in the spinal region as was noted by Desai et al ${ }^{[4]}$ who found four out of 6 intraspinal cases to be psammomatous. In our study, four out of 7 intraspinal cases were psammomatous meningiomas. Majority of the cases (four) presented in 4th decade of life. Out of the 10 cases, 8 were female and two were male.

There were 6 cases of angiomatous meningioma (9.52\%) out of total 63 cases in our study. Angiomatous meningioma is rare comprising about $2.1 \%$ of all meningiomas ${ }^{[19]}$. In the study done by Desai et at ${ }^{[4]}$, angiomatous meningioma amounted to $8 \%$ of all meningiomas, which were in concordance with our study. All the 6 angiomatous cases were located intracranially, out of which four were in cerebral convexity.

Fibrous meningioma constituted of 2 cases (3.17\%) out of 63 cases in our study. Various studies in literature showed the percentage of fibrous meningioma to be in the range of $4.8-16 \%{ }^{[10,20]}$. We encountered one case each of microcystic and secretory meningioma (1.58\%) out of total 63 cases. Microcystic meningiomas are also rare and the results in our study were comparable to the studies conducted by Gadgil et al ${ }^{[17]}$ and Shrilakshmi et al ${ }^{[12]}$ who found $0.6 \%$ and $0.78 \%$ of microcystic variant out of all meningiomas respectively. The incidence of secretory meningioma ranges from $1.2-9.3 \%$ in the literature ${ }^{[21]}$. These accounted for $1.35 \%$ in the study done by Wang et al ${ }^{[22]}$ which were in concordance with our study. We did not find any Lymphoplasmacyte-rich and Metaplastic variant during our study period.

Grade II meningioma comprised of 7 cases out of total 63 meningioma cases in our study $(11.11 \%)$. This is in concordance with the study conducted by Gadgil et al ${ }^{[17]}$ and Shah et al ${ }^{[10]}$ where the incidence of grade II meningioma was $11.5 \%$ and $8 \%$ respectively. The studies done by Samadi et al ${ }^{[20]}$ demonstrated a male predominance in grade II meningioma, indicating increased risk of male gender for grade II meningioma. However, results in our study are in contradiction to those found in this study. This variation could be due to less sample size in our study, genetic or environmental factors ${ }^{[20]}$.

Grade II comprised of 5 cases of atypical meningioma and two cases of chordoid meningioma. Two cases of chordoid meningioma $(3.17 \%)$ were female patients who presented in 5th and 6th decade of life. Our results were comparable to the study done by Samadi et al ${ }^{[20]}$ where the percentage of chordoid meningioma was $1.3 \%$. Among the 5 atypical meningiomas, high mitotic count was the most commonly applied parameter for categorizing meningioma as grade II; mitotic count being more than 4 per 10 high power field in three cases. The other two cases of atypical meningioma showed presence of brain invasion, categorizing these as grade II meningioma. Hypercellularity, macronucleoli, high nucleus: cytoplasm ratio and necrosis were also seen in many cases out of the 63 studied cases of meningioma, but due to lack of presence of 3 or more features in individual case, these failed to classify as grade II and thus belonged to grade I meningioma.

Mitotic count is an important criterion for determining a meningioma as grade II. Mitotic rate was counted per 10 high power fields in the areas having highest mitotic figures. We found mitosis in 18 cases out of total 63 cases (28.57\%). Though mitosis was seen in 18 cases, Only 3 cases demonstrated mitotic rate of more than 4 per 10 high power fields, and thus were graded as meningioma grade II. Counting of mitotic figures is sometimes difficult due to various factors. The density of mitotic activity varies in different areas. Mitotic activity is usually counted in the areas having highest mitotic figures. There may be interobserver variability in choosing such areas for mitotic count. Sometimes, it may be difficult to differentiate mitotic figures from pyknotic nuclei or from apoptotic bodies. Immunohistochemical staining specific for phosphorylated histone $\mathrm{H} 3$ ( $\mathrm{PHH} 3$ ) has been noted to improve the efficiency in counting the mitotic figures 
[23]. Histone $\mathrm{H} 3$ yields maximum phosphorylation during mitosis but is demonstrated to remain unphosphorylated during apoptosis. This staining technique may help in identifying mitotic figures, but has not become a routine practice.

Brain invasion was seen in two cases out of total 63 cases of meningioma $(3.17 \%)$. Brain invasion is characterized by the presence of irregular tongue like protrusion of tumour cells infiltrating the underlying brain parenchyma without an intervening layer of leptomeninges. Initially brain invasion was not considered as a separate histological parameter for grade II meningiomas. But various studies ${ }^{[3]}$ established the prognosis of meningioma with brain invasion to be similar to grade II and grade III meningiomas. Hence, brain invasion is used as a separate histological parameter for grade II. Even if histological features are that of benign meningioma, the presence of brain invasion warrants a classification of grade II meningioma.

Psammoma bodies were seen in 28 cases out of total 63 cases of meningioma (44.44\%). The only cases which showed predominance of psammoma bodies over that of meningothelial components were categorized as psammomatous meningioma. Some studies noted that the presence of psammoma bodies have a protective prognostic value.

Necrosis may occur focally or in large areas. Necrosis occurring due to preoperative embolization or radiation should not be considered; rather only spontaneous necrosis should be accounted while diagnosing meningioma. Macronucleoli are said to be present if the nucleoli are visualized at $10 \mathrm{x}$ power. Necrosis, hypercellularity, high nucleus: cytoplasm ratio and macronucleoli are the parameters used for the diagnosis of grade II meningioma when a minimum of these three features are present together. Many of our cases showed one or two of these features and hence were categorized as grade I meningioma.

Although female predominance is seen in meningioma overall, some studies in literature showed that male gender is associated with more risk of atypical and anaplastic meningioma ${ }^{[20,24]}$. This could be due to difference in hormone level and hormone receptor status. There is evidence which demonstrate more progesterone receptor expression in benign meningiomas than in higher grade ${ }^{[25]}$. Also, there is an inverse relationship between progesterone receptor expression and WHO tumour grade of meningioma. All these factors may suggest male gender as a risk factor for grade II and III meningioma. However, this was not demonstrated this in our study.

An important role of immunohistochemistry in meningioma diagnostics lies in the valuation of the proliferative index.
Along with the histological parameters, proliferation indices play an imperative role in predicting the behaviour of meningioma. Amongst the various proliferation indices available to measure cell proliferation, Ki67 is the most commonly used immunohistochemical marker.

Ours being a tertiary care hospital, patients were primarily referred here for surgery and further ancillary treatment was carried out at or near their hometown. IHC was not available at our institute and hence patients were referred for IHC to the oncology institution. Ancillary treatment and follow-up were done at the same institute.

\section{Conclusion}

Meningiomas presents with a variety of clinical features. Therefore, radiology and histopathology are mandatory for reaching a final diagnosis which is necessary for the proper management of the patient. Radiologically, meningioma shows characteristic finding and is thus extremely helpful modality in the management process of the patient. However, the presence of diverse histological variants, grades and the associated prognosis warrants the use of histopathological examination as an important and obligatory tool for the final diagnosis of this lesion. The current grading system is based on histological features and is of prognostic importance. The use of ancillary technique like immunohistochemistry aids in the course of diagnosis. A continual revision of the histopathology of meningioma is necessary to improve the accuracy and reproducibility of the histopathological diagnosis and grading of these tumours.

\section{Reference}

1. Marosi C, Hassler M, Roessler K, et al. Meningioma. Crit Rev Oncol Hematol. 2008;67(2):153-171. doi:10.1016/j. critrevonc.2008.01.010.

2. Riemenschneider MJ, Perry A, Reifenberger G. Histological classification and molecular genetics of meningiomas. Lancet Neurol. 2006;5(12):1045-1054. doi:10.1016/S14744422(06)70625-1.

3. Louis DN, Ohgaki H, Wiestler OD, Cavenee WK. WHO Classification of Tumours of the Central Nervous System. IARC: Lyon.; 2007.

4. Desai P, Patel D. Histopathological study of meningioma. Int J Med Sci Public Heal. 2016;5(2):327-330. doi:10.5455/ ijmsph.2016.04102015125.

5. Baumgartner JE, Sorenson JM. Meningioma in the pediatric population. J Neurooncol. 1996;29:223-228. doi:10.1007/ BF00165652.

6. Backer-Grøndahl T, Moen BH, Torp SH. The histopathological spectrum of human meningiomas. Int $\mathrm{J}$ Clin Exp Pathol. 2012;5(3):231-242. 
7. Wahab M, Al-Azzawi F. Meningioma and hormonal influences. Climacteric. 2003;6(4):285-292. doi:10.1080/ cmt.6.4.285.292.

8. Galgano M, Beutler T, Brooking A, Deshaies E. Spinal Meningiomas: A Review. J Spine. 2014;3(1):157. doi:10.4172/2165-7939.1000157.

9. Moradi A, Semnani V, Djam H, et al. Pathodiagnostic parameters for meningioma grading. J Clin Neurosci. 2008;15(12):1370-1375. doi:10.1016/j.jocn.2007.12.005.

10. Shah S, Gonsai RN, Makwana R. HISTOPATHOLOGICAL STUDY OF MENINGIOMA IN CIVIL HOSPITAL , AHMEDABAD. Int J Curr Res Rev. 2013;5(3):76-82.

11. Gangadhar K, Santhosh D, Gm F. Imaging Features of Intracranial Meningiomas with Histopathological Correlation: A Relook into Old Disease. Nepal J Radiol. 2013;3:1(4):14-32.

12. Lakshmi SS. Meningiomas: A Clinicopathological study. Int J Med Res Heal Sci. 2015;4(4):827-831. doi:10.5958/23195886.2015.00164.2.

13. Patil P, Patil PR, Sondankar D. Clinicopathological Study of Meningioma. Int J Med Res Rev. 2016;4(4):592-601. http://medresearch.in/index.php/IJMRR/article/view/551. Accessed December 23, 2016.

14. Deb P, Sahani H, Bhatoe HS, Srinivas V. Intraventricular cystic meningioma. J Cancer Res Ther. 2010;6(2):218-220. doi:10.4103/0973-1482.65247.

15. Mary AK, Mary AK, Abuya JM, Chumba D, Koech FK. The Common Radiological Features of Meningiomas on CT scan and MRI among Patients at Major Hospitals in Eldoret, Kenya. 2013;2(2):213-226.

16. Kim BW, Kim MS, Kim SW, Chang $\mathrm{CH}$, Kim OL. Peritumoral brain edema in meningiomas: Correlation of radiologic and pathologic features. J Korean Neurosurg Soc. 2011;49(1):26-30. doi:10.3340/jkns.2011.49.1.26.
17. Gadgil NM, Margam SR, Chaudhari CS, Kumavat PV. The histopathological spectrum of meningeal neoplasms. Indian J Pathol Oncol. 2016;3(3):432-436. doi:10.5958/23946792.2016.00081.8.

18. Jindal A, Choudhary S. A Clinicopathological Study of Meningioma with Special Reference on Variants and Grading in a Tertiary Care Centre. Int J Med Res Prof. 2016;2(3):192-196. doi:10.21276/ijmrp.2016.2.3.042.

19. Hasselblatt M, Nolte KW, Paulus W. Angiomatous meningioma: a clinicopathologic study of 38 cases. Am J Surg Pathol. 2004;28(3):390-393. http://www.ncbi.nlm.nih. gov/pubmed/15104303. Accessed December 23, 2016.

20. Samadi N, Ahmadi S. Meningioma: A clinicopathological evaluation. Malaysian J Med Sci. 2007;14(1):46-52. doi:10.2337/db06-1182.J.-W.Y.

21. Regelsberger J, Hagel C, Emami P, Ries T, Heese O, Westphal M. Secretory meningiomas: a benign subgroup causing lifethreatening complications. Neuro Oncol. 2009;11:819-824. doi:10.1215/15228517-2008-109.

22. Wang DJ, Xie Q, Gong Y, et al. Secretory meningiomas: Clinical, radiological and pathological findings in 70 consecutive cases at one institution. Int J Clin Exp Pathol. 2013;6(3):358-374.

23. Commins DL, Atkinson RD, Burnett MD. Review of meningioma histopathology. Neurosurg Focus. 2007;23(4):1-9. doi:10.3171/foc.2007.23.4.4.

24. Kane A, Sughrue M, Rutkowski M, et al. Anatomic location is a risk factor for Atypical and Malignant meningiomas. Cancer. 2011;117(6):1272-1278. doi:10.1097/ OPX.0b013e3182540562.The.

25. Taghipour M, Rakei SM, Monabati A. The role of estrogen and progesterone receptors in grading of the malignancy of meningioma. Iran Red Crescent Med J. 2007;9(1):17-21.

*Corresponding author:

Dr. Shalaka Khade, 499, Raghukul-8, Lendra Park, Ramdaspeth, Nagpur-440010, India

Phone: +91 8600364846

Email: shalakakhade18@gmail.com

Financial or other Competing Interests: None. 